\title{
Generation of CsI Cluster Ions for Mass Calibration in Matrix-Assisted Laser Desorption/Ionization Mass Spectrometry
}

\author{
Xianwen Lou, Joost L. J. van Dongen, and E. W. Meijer \\ Laboratory of Macromolecular and Organic Chemistry, Eindhoven University of Technology, Eindhoven, \\ The Netherlands
}

A simple method was developed for the generation of cesium iodide (CsI) cluster ions up to $\mathrm{m} / \mathrm{z}$ over 20,000 in matrix-assisted laser desorption/ionization mass spectrometry (MALDI MS). Calibration ions in both positive and negative ion modes can readily be generated from a single MALDI spot of $\mathrm{CsI}_{3}$ with 2-[(2E)-3-(4-tert-butylphenyl)-2-methylprop-2-enylidene] malononitrile (DCTB) matrix. The major cluster ion series observed in the positive ion mode is $\left[(\mathrm{CsI})_{\mathrm{n}} \mathrm{Cs}\right]^{+}$, and in the negative ion mode is $\left[(\mathrm{CsI})_{\mathrm{n}} \mathrm{I}\right]^{-}$. In both cluster series, ions spread evenly every 259.81 units. The easy method described here for the production of CsI cluster ions should be useful for MALDI MS calibrations. (J Am Soc Mass Spectrom 2010, 21, 1223-1226) (c) 2010 American Society for Mass Spectrometry

A central issue in mass spectrometry (MS) analysis is to provide molecular weight information of analytes. The degree of mass accuracy largely depends on the instrument type and mass calibration method employed. Nowadays, accurate mass measurements with mass error of only a few parts per million (ppm) can be made using a variety of instrument types [1-5]. High mass accuracy is extremely important in the MS application of molecular formula determination and can significantly improve the level of confidence for compound identification, such as protein/peptide confirmation, through database searching [6-10].

For accurate mass measurements, a MS system must be precisely calibrated. Calibration constants are usually determined by measuring several reference ions covering the mass range of interest. Different calibration methods have been developed in matrix-assisted laser desorption/ionization (MALDI) MS aiming to improve the mass accuracy [11-18]. Nevertheless, a crucial step in calibration remains to be the generation of suitable ions. In principle, any compounds that can yield ions with known masses and do not interfere with the MS analysis might be applied as calibration standards. In MALDI MS, widely used standards include peptides, proteins, and synthetic polymers/oligomers. To establish a precise calibration, a number of standards spreading over the mass range of interest should be included. Because of the analyte suppression effect (ASE) in MALDI $[19,20]$, some analyte/calibrant ions of interest might substantially or even completely be suppressed. As a consequence, the preparation of suitable

Address reprint requests to Dr. X. W. Lou, Laboratory of Macromolecular and Organic Chemistry, Eindhoven University of Technology, P.O. Box 513, 5600 MB Eindhoven, The Netherlands. E-mail: x.w.lou@tue.nl calibrants in MALDI, even for external calibration, is not a simple task and may involve an extensive trial and error procedure. Furthermore, different calibration standards are generally required for different modes of ionization (positive or negative ion modes) and for different mass ranges of interest. In fact, MALDI MS still suffers from the lack of truly convenient mass standard calibrants.

In this contribution, we described a convenient method of generating cesium iodide (CsI) cluster ions for MALDI MS calibration. CsI cluster ions, together with a number of other cluster ions, have frequently been used in electrospray ionization (ESI) MS calibration. Under suitable ESI conditions, cluster ions evenly covering a broad $\mathrm{m} / \mathrm{z}$ range can be recorded. Calibration curves thus made have many data points and will generally provide a very precise calibration [21-24]. Compared with other types of cluster ions, the CsI cluster ions are quite unique because both Cs and I are monoisotopic. Evidently, the monoisotopic property is desirable in MS calibration where the mass accuracy of peak centroids of the calibrants might otherwise be impaired by the skewed/asymmetric peak shape due to their unresolved isotopic distributions. Another important advantage of CsI cluster ions is that they can be generated in both positive and negative ion modes. These properties of CsI cluster ions also make them very attractive to be used for MALDI MS calibrations. $\mathrm{O}^{\prime}$ Connor and Costello reported the application of CsI clusters for the internal calibration of Fourier transform mass spectrometry (FTMS) with mass accuracy of $<1$ ppm [13]. However, in their report only small clusters up to $\mathrm{Cs}_{4} \mathrm{I}_{3}$ were observed. In this study, a simple method is described to generate CsI cluster ions up to 
mass over 20,000 Da in both positive and negative ion modes in MALDI MS.

\section{Experimental}

The MALDI TOF MS measurements were performed with a Voyager-DE Pro (PerSeptive Biosystems, Framingham, MA, USA) instrument equipped with a 337 $\mathrm{nm}$ nitrogen laser with 3 ns-duration pulse, capable of executing both linear and reflector modes. In the reflector mode, the accelerating voltage was held at $20 \mathrm{kV}$ and delay time at 350 ns, while in the linear mode the accelerating voltage at $25 \mathrm{kV}$ and the delay time at 400 ns. Mass spectra were acquired by summing spectra from 100 selected laser shots.

$\mathrm{CsI}_{3}, \mathrm{CsI}$, and $\mathrm{N}(\mathrm{Bu})_{4} \mathrm{I}_{3}$ (tetrabutylammonium triiodide) were purchased from Aldrich (Zwijndrecht, The Netherlands). Three matrices were used in this work. 2-[(2E)-3-(4-tert-butylphenyl)-2-methylprop-2-enylidene] malononitrile (DCTB) were synthesized according to Ulmer et al. [25], and $\alpha$-cyano-4-hydroxycinnamic acid (CHCA) and 2,5-dihydroxybenzoic acid (DHB) were obtained from Fluka (Buchs, Switzerland). Matrix solutions were freshly prepared in tetrahydrofuran (THF) at concentrations of about $100 \mu \mathrm{mol} / \mathrm{mL}$. A matrix solution and a sample solution (also in THF) were combined in an Eppendorf tube and vortex-mixed for at least $10 \mathrm{~s}$, and about $0.5 \mu \mathrm{L}$ of the mixed solution was pipetted onto a stainless steel MALDI target plate.

\section{Results and Discussion}

In ESI MS, CsI cluster ions can readily be generated by electrospraying a CsI solution at an appropriate concentration. When CsI was tried in MALDI TOF MS, however, no such ions were observed. Fortunately, by simply replacing $\mathrm{CsI}$ with $\mathrm{CsI}_{3}$, abundant $\mathrm{CsI}$ cluster ions could easily be generated in MALDI MS as well.

Figure 1 shows MALDI TOF MS spectra of $\mathrm{CsI}_{3}$ with DCTB matrix in the linear mode. CsI cluster species containing singly charged ions every 259.81 Da up to $\mathrm{m} / \mathrm{z}$ over 20,000 were observed in both positive and negative ion modes. The major cluster ion series observed in the positive ion mode is $\left[(\mathrm{CsI})_{n} \mathrm{Cs}\right]^{+}$, and in the negative ion mode $\left[(\mathrm{CsI})_{\mathrm{n}} \mathrm{I}\right]^{-}$. In addition, cluster ion series of $\left[(\mathrm{CsI})_{n} \mathrm{Cs}+\mathrm{NaI}^{+}\right.$and $\left[(\mathrm{CsI})_{n} \mathrm{I}+\mathrm{NaI}^{-}\right.$with much less signal intensity were also observed in the positive and negative ion modes, respectively. In Figure 1 , the molar ratio of $\mathrm{CSI}_{3} / \mathrm{DCTB}$ is $1 / 2$, and similar results were observed in the molar ratio range of $1 / 1$ to $1 / 10$. It should be noted here that the $\mathrm{CsI}_{3} / \mathrm{DCTB}$ ratios applied are quite high compared with the typical analyte/matrix values in normal MALDI TOF MS measurements. Although cluster ions could also be detected at much lower $\mathrm{CsI}_{3}$ /DCTB ratios, the molecular weight of the biggest cluster ions recorded will decrease dramatically with decreased $\mathrm{CsI}_{3} / \mathrm{DCTB}$ ratios. The requirement of a high $\mathrm{CsI}_{3} / \mathrm{DCTB}$ ratio is probably because the high ratio will favor the formation of large cluster ions.

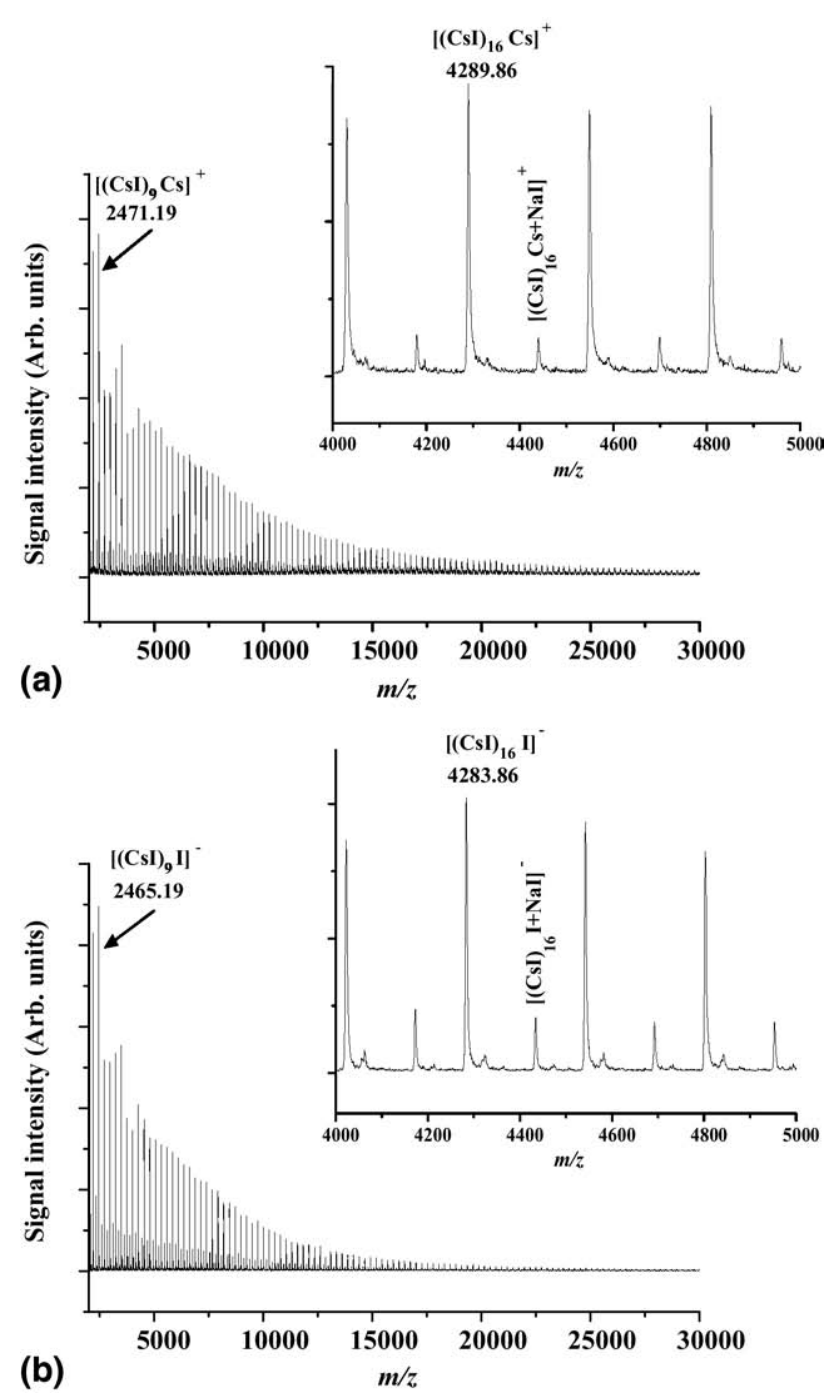

Figure 1. MALDI TOF MS spectra of $\mathrm{CsI}_{3}$ with DCTB matrix in the linear mode. The molar ratio of $\mathrm{CsI}_{3} / \mathrm{DCTB}$ is 1:2. (a) Linear positive ion mode; (b) linear negative ion mode.

On the other hand, it is interesting to see that even direct laser desorption/ionization (LDI) of $\mathrm{CsI}_{3}$ without matrix could yield cluster ions up to $m / z$ of about 8000 . This value is, however, significantly lower than that observed in Figure 1. By comparing the results of LDI and MALDI, it seems that DCTB matrix at a suitable $\mathrm{CsI}_{3} / \mathrm{DCTB}$ ratio would facilitate the formation of larger CsI cluster ions.

In addition to being observed in the linear mode, both positive and negative CsI cluster ions could also be recorded in the reflector mode. Figure 2 shows MALDI TOF MS spectra of $\mathrm{CsI}_{3}$ with DCTB matrix in the reflector mode. Cluster ions of $\mathrm{m} / \mathrm{z}$ around 3500 can clearly be observed.

Although CsI cluster ions up to $m / z$ over 20,000 could be generated in MALDI using $\mathrm{CsI}_{3}$ with DCTB as the matrix, it is unclear how these ions were formed. In contrast to the observation of abundant CsI cluster ions, no $\mathrm{CsI}_{3}$ clusters were observed in the positive ion mode 


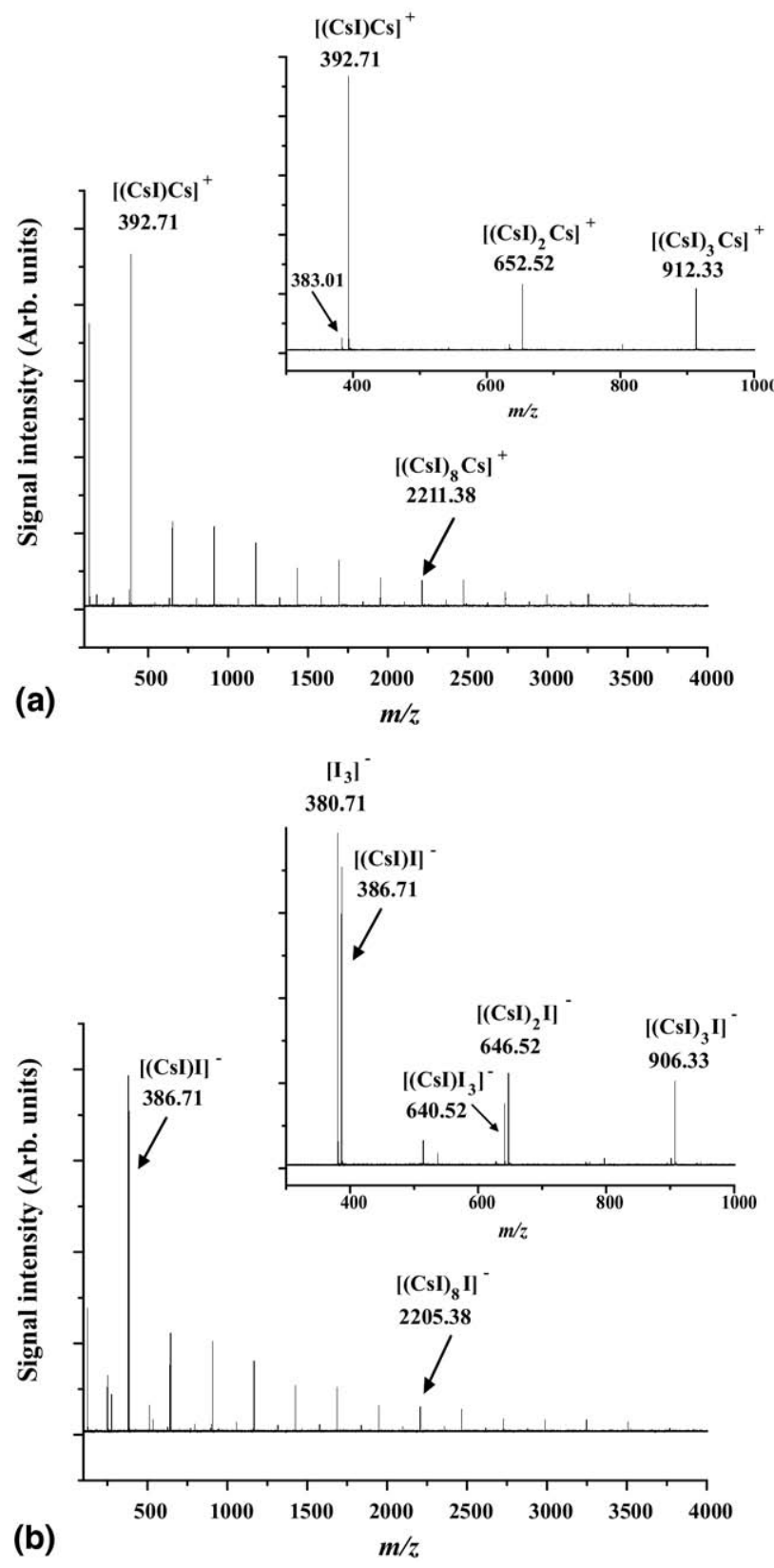

Figure 2. MALDI TOF MS spectra of $\mathrm{CsI}_{3}$ with DCTB matrix in the reflector mode. The molar ratio of $\mathrm{CsI}_{3} / \mathrm{DCTB}$ is 1:2. (a) Reflector positive ion mode; (b) reflector negative ion mode.

despite of the excessive amount of $\mathrm{CsI}_{3}$ present in the MALDI sample (see Figure 2a). Similar results were also obtained in the negative ion mode. Concerning the formation of $\mathrm{CsI}_{3}$ clusters and/or $\left[\mathrm{I}_{3}\right]^{-}$adducts, only $\left[\mathrm{I}_{3}\right]^{-}$and a small adduct of $\left[(\mathrm{CsI}) \mathrm{I}_{3}\right]^{-}$were detected with strong signal intensity, and no $\left[\mathrm{I}_{3}\right]^{-}$adducts with ion mass higher than $\left[(\mathrm{CsI})_{2} \mathrm{I}_{3}\right]^{-}$were recorded (see Figure $2 b$ ). Furthermore, when CsI was used directly instead of $\mathrm{CsI}_{3}$, no CsI cluster ions were detected. This implies that $\left[\mathrm{I}_{3}\right]^{-}$might play an important role in CsI cluster ion formation. However, it was found that not all triiodides could give analyte cluster ions because no corresponding cluster ions were observed when another triiodide compound (tetrabutylammonium triiodide $\left.\left(\mathrm{N}(\mathrm{Bu})_{4} \mathrm{I}_{3}\right)\right)$ was used instead of $\mathrm{CsI}_{3}$.

Besides of DCTB, two other matrices, 2,5dihydroxybenzoic acid (DHB) and $\alpha$-cyanno-4-hydroxycynnamic acid (CHCA) were also studied. Contrary to what was observed with DCTB matrix, very weak and only low molecular weight CsI cluster ions were observed with DHB while negligible or no such cluster ions at all with CHCA. The results clearly indicate that the formation of CsI cluster ions is strongly matrix-dependent.

Because of the lack of CsI cluster ions with some matrices, an immediate question is whether the CsI cluster ions generated with, for example, DCTB matrix can be applied for the calibration of MALDI MS measurements with other matrices. A major concern of this question is the matrix-dependent behavior of the initial velocity of MALDI ions [26, 27]. In MALDI MS calibrations, the experimental conditions and the instrument design should be calibrated with the reference ions. The influence of ion initial velocity on the accuracy of calibration relies on which type of mass analyzer is coupled to MALDI. For such MALDI MS combinations as MALDI ion-trap, MALDI Fourier transfer ion cyclotron resonance (FT-ICR) and MALDI orthogonal acceleration (oa) time-of-flight (TOF), the variation of ion initial velocity shows no influence on calibration or the influence can be corrected satisfactorily. Under these circumstances, the strong CsI cluster ions produced using DCTB matrix can be employed to calibrate a MS system or MALDI MS measurements without considering the nature of the matrix employed. However, if MALDI is coupled with axial TOF, which is still the most popular type of MALDI MS combination, the influence of ion initial velocity on the calibration accuracy must be considered. The initial velocities of MALDI ions are generally less than $1000 \mathrm{~m} / \mathrm{s}$, and mostly in the range of $300-600 \mathrm{~m} / \mathrm{s}[26,27]$. The effects of variation/dispersion of ion initial velocity on ion fight time can largely be corrected in a delayed extraction and by a reflector (in the reflector mode) [28-31]. Therefore, in the delayed reflector mode, the calibration error introduced by using different matrices can significantly be minimized and might be tolerable in many MALDI MS applications. In our laboratory, the CsI cluster ions produced using DCTB matrix were frequently employed for the external calibration of MALDI TOF MS measurements with CHCA and DHB matrices in the delayed reflector mode, and the mass accuracies obtained were well within our instrument specification $(0.01 \%$ with external calibration in the delayed reflector mode) [32]. Based on the discussions above, it can be concluded that the easy method described here for the generation of both positive and negative CsI cluster ions should be useful for the calibration of MALDI MS measurements. 


\section{References}

1. Bristow, A. W. T. Accurate Mass Measurement for the Determination of Elemental Formula-A Tutorial. Mass Spectrom. Rev. 2006, 215, 99-111.

2. Marshall, A. G.; Hendrickson, C. L.; Shi, S. D.-H. Scaling MS Plateaus with High Resolution FT-ICRMS. Anal. Chem. 2002, 74, 253A-259A.

3. Williams, J. D.; Flanagan, M.; Lopez, L.; Fischer, S.; Miller, L. A. D. Using Accurate Mass Electrospray Ionization-Time-of-Flight Mass Spectrometry with In-source Collision-Induced Dissociation to Sequence Peptide Mixtures. J. Chromtogr. A 2003, 1020, 11-26.

4. Bristow, A. W. T.; Webb, K. S. Inter-Comparison Study on Accurate Mass Measurement of Small Molecules in Mass Spectrometry. J. Am. Soc. Mass Spectrom. 2003, 14, 1086-1098

5. Russell, D. H.; Edmondson, R. D. High-Resolution Mass Spectrometry and Accurate Mass Measurements with Emphasis on the Characterization of Peptides and Proteins by Matrix-Assisted Laser Desorption/ Ionization Time-of-Flight Mass Spectrometry. J. Mass Spectrom. 1997, 32, 263-276.

6. Beynon, J. H. Qualitative Analysis of Organic Compounds by Mass Spectrometry. Nature 1954, 174, 735-737.

7. Yates, J. R. III Database Searching Using Mass Spectrometry Data. Electrophoresis 1998, 19, 893-900.

8. Witt, M.; Fuchser, J.; Baykut, G. Fourier Transform Ion Cyclotron Resonance Mass Spectrometry with NanoLC/Microelectrospray Ionization and Matrix-Assisted Laser Desorption/Ionization: Analytical Performance in Peptide Mass Fingerprint Analysis. J. Am. Soc. Mass Spectrom. 2003, 14, 553-561.

9. Thurman, E. M.; Ferrer, I.; Fernandez-Alba, A. R. Matching Unknown Empirical Formulas to Chemical Structure Using LC/MS TOF Accurate Mass and Database Searching: Example of Unknown Pesticides on Tomato Skins. J. Chromatogr. A 2005, 1067, 127-134.

10. Schlosser, A.; Lehmann, W. D. Patchwork Peptide Sequencing: Extraction of Sequence Information from Accurate Mass Data of Peptide Tandem Mass Spectra Recorded at High Resolution. Proteomics 2002, 2, 524-533.

11. Vera, C. C.; Zubarev, R.; Ehring, H.; Hankansson, P.; Sunqvist, B. U. R. A Three-Point Calibration Procedure for Matrix-Assisted Laser Desorption/Ionization Mass Spectrometry Utilizing Multiply Charged Ions and Their Mean Initial Velocities. Rapid Commun. Mass Spectrom. 1996, 10, 1429-1432.

12. Christian, N. P.; Arnold, R. J.; Reilly, J. P. Improved Calibration of Time-of-Flight Mass Spectrometry by Simplex Optimization of Electrostatic Ion Calculations. Anal. Chem. 2000, 72, 3327-3337.

13. O'Connor, P. B.; Costello, C. E. Internal Calibration on Adjacent Samples (InCAS) with Fourier Transform Mass Spectrometry. Anal. Chem. 2000, 72, 5881-5885.

14. Gobom, J.; Mueller, M.; Egelhofer, V.; Theiss, D.; Lehrach, H.; Nordhoff, E. A Calibration Method That Simplifies and Improves Accurate Determination of Peptide Molecular Masses by MALDI-TOF MS. Anal. Chem. 2002, 74, 3915-1923.

15. Hack, C. A.; Benner, W. H. A Simple Algorithm Improves Mass Accuracy to 50-100 ppm for Delayed Extraction Linear Matrix-Assisted Laser Desorption/Ionization Time-of-Fight Mass Spectrometry. Rapid Commun. Mass Spectrom. 2002, 16, 1304-1312.
16. Moskovets, E.; Karger, B. L. Mass Calibration of a Matrix-Assisted Laser Desorption/Ionization Time-of-Flight Mass Spectrometry Including the Rise Time of the Delayed Extraction Pulse. Rapid Commun. Mass Spectrom. 2003, 17, 229-237.

17. Moskovets, E.; Chen, H.-S.; Pashkova, A.; Rejtar, T.; Andreev, V.; Karger, B. L. Closely Spaced External Standard: A Universal Method of Achieving 5 ppm Mass Accuracy Over the Entire MALDI plate in Axial Matrix-Assisted Laser Desorption/Ionization Time-of-Fight Mass Spectrometry. Rapid Commun. Mass Spectrom. 2003, 17, 2177-2187.

18. Vestal, M. L. Modern MALDI Time-of-Fight Mass Spectrometry. J. Mass Spectrom. 2009, 44, 303-317.

19. Knochenmuss, R.; Stortelder, A.; Breuker, K.; Zenobi, R. Secondary Ion-Molecule Reactions in Matrix-Assisted Laser Desorption/Ionization. J. Mass Spectrom. 2000, 35, 1237-1245.

20. Knochenmuss, R.; Zenobi, R. MALDI. Ionization: The Role of In-Plume Processes. Chem. Rev. 2003, 103, 441-452.

21. Pleasance, S.; Thibault, P.; Sim, P. G.; Boyd, R. K. Cesium Iodide Clusters as Mass Calibrants in Ionspray Mass Spectrometry. Rapid Commun. Mass Spectrom. 1991, 5, 307-308.

22. Stout, S. J.; daCunha, A. R. Tuning and Calibration in Thermospray Liquid Chromatography/Mass Spectrometry Using Trifluoroacetic Acid Cluster Ions. Anal. Chem. 1989, 61, 2126-2128.

23. Hop, C. E. C. A. Generation of High Molecular Weight Cluster Ions by Electrospray Ionization: Implications for Mass Calibration. J. Mass Spectrom. 1996, 31, 1314-1316.

24. Ledman, D. W.; Fox, R. O. Water Cluster Calibration Reduces Mass Error in Electrospray Ionization Mass Spectrometry of Proteins. J. Am. Soc. Mass Spectrom. 1997, 8, 1158-1164.

25. Ulmer, L.; Torres-Gacia, H. G.; Mattay, J.; Luftmann, H. The Use of 2-[(2E)-3-(4-tert-Butylphenyl)-2-Methylprop-2-Enylidene]Malononitrile as Matrix for Matrix-Assisted Laser Desorption/Ionization Mass Spectrometry. Eur. J. Mass Spectrom. 2000, 6, 49-52.

26. Gluckmann, M.; Karas, M. The Initial Ion Velocity and Its Dependence on Matrix, Analyte, and Preparation Method in Ultraviolet MatrixAssisted Laser Desorption Ionization. J. Mass Spectrom. 1999, 34, 467477.

27. Karas, M.; Kruger, R. Ion Formation in MALDI: The Cluster Ionization Mechanism. Chem. Rev. 2003, 103, 427-439.

28. Whittal, R. M.; Li, L. High-Resolution Matrix-Assisted Laser Desorption/Ionization in a Linear Time-of-Flight Mass Spectrometry. Anal. Chem. 1995, 67, 1950-1954.

29. Juhasz, P.; Vestal, M. L.; Martin, S. A. On the Initial Velocity of Ions Generated by Matrix-Assisted Laser Desorption Ionization and Its Effect on the Calibration of Delayed Extraction Time-of-Flight Mass Spectrometry. J. Am. Soc. Mass Spectrom. 1997, 8, 209-217.

30. Vestal, M.; Juhasz, P. Resolution and Mass Accuracy in Matrix-Assisted Laser Desorption Ionization Time-of-Flight. J. Am. Soc. Mass Spectrom. 1998, 9, 892-911.

31. Edmondson, R. D.; Russell, D. H. Evaluation of Matrix-Assisted Laser Desorption Ionization Time-of-Flight Mass Measurement Accuracy by Using Delayed Extraction. J. Am. Soc. Mass Spectrom. 1996, 7, 995-1001.

32. User Guide of Voyager Biospectrometry Workstation, Applied Biosystems, Version 5.1, p. A-5. 